\title{
CONHECIMENTO SOBRE 0 DIAGNÓSTICO E RASTREAMENTO DO CÂNCER DE MAMA ENTRE OS GINECOLOGISTAS DO ESTADO DE GOIÁS (BRASIL)
}

\author{
Ruffo de Freitas Júnior*, Evelling lorena Cerqueira de Oliveira, Eneida Ribeiro Marinho, \\ Rossana Araújo Catão Zampronha, Rubens José Pereira, francisco Aquino Soares \\ Trabalho realizado no Programa de Mastologia do Departamento de Ginecologia e Obstetrícia \\ da Faculdade de Medicina da Universidade Federal de Goiás e Serviço de Ginecologia e Mama \\ do Hospital Araújo Jorge da Associação de Combate ao Câncer em Goiás, GO.
}

RESUMO - OBjetivos. Avaliar o nível de conhecimento sobre o rastreamento e diagnóstico do câncer de mama entre os ginecologistas do Estado de Goiás, Brasil.

Métodos. Foi enviado um questionário contendo perguntas sobre a formação profissional e sobre o diagnóstico do câncer de mama, para os 427 sócios da Sociedade Goiana de Ginecologia e Obstetrícia, entre maio e julho de 1999.

Resultados. Dos 427 questionários enviados, 26 (6\%) foram devolvidos por endereçamento incorreto. Dos $40 \mathrm{I}$ médicos que receberam o questionário, $85(21 \%)$ responderam e retornaram. Destes, $28 \%$ eram do sexo feminino, a média do tempo de profissão foi de 17 anos e $70 \%$ possuía a residência médica como maior titularidade. A média de idade foi de 44 anos. As taxas de respostas corretas foram: $94 \%$ sabiam o sinal mais freqüente do câncer de mama, $75 \%$ o melhor método para rastreamento, $88 \%$ o período de quando iniciar a mamografia, $93 \%$ a periodicidade da mamografia após os 50 anos e $83 \%$ sobre a indicação da ultra-sonografia complementar. $O$ fato de o profissional ter cursado um programa de Residência Médica foi o único fator que influenciou na taxa de respostas corretas, se comparado àqueles que não cursaram. 0 sexo do profissional, portadores de Título de Especialista em ginecologia e obstetrícia (TEGO) e a cidade de atuação não influenciaram nas taxas de respostas corretas.

Conclusōes. Os ginecologistas do Estado de Goiás que responderam ao questionário têm um bom conhecimento sobre 0 rastreamento e diagnóstico do câncer de mama, sendo que apenas a Residência Médica influenciou na taxa de respostas corretas.

UnITERMOS. Mama. Câncer. Diagnóstico. Ensino médico. Ginecologistas.

\section{INTRODUÇÃO}

O câncer de mama tem sido motivo crescente de preocupação, com uma tendência mundial de aumento da sua incidência ${ }^{1-4}$. Para a região Centro-oeste, estima-se 36 novos casos de câncer de mama para 100.000 muIheres no ano de 200I, com uma taxa de mortalidade de 9/100.000. Assim, devem ter sido diagnosticados, na região Centro-Oeste, 880 novos casos de câncer de mama para o mesmo ano, sendo 150 desses na cidade de Goiânia ${ }^{2,4}$.

O diagnóstico precoce do câncer de mama é um importante fator na escolha do tratamento adequado, na mortalidade e na expectativa de vida da paciente ${ }^{5-9}$. Mesmo detendo esse conhecimento, em alguns locais do país, aproximadamente dois terços dos casos de cânceres da mama são diagnosticados nos estádios avançados da doença ${ }^{10-12}$

\footnotetext{
* Correspondência:

Av. T-4, n 835, Ap. 901, Setor Bueno

74230-030 - Goiânia - GO - ruffo@medicina.ufg.br Tel/Fax: (062) 243-7244
}

e a principal causa deste quadro parece ser a desinformação da população frente ao problema ${ }^{8}$.

Nos países desenvolvidos, programas populacionais de detecção precoce do câncer de mama (programas de rastreamento), com mamógrafos de alta resolução, têm oferecido alguns pontos importantes: aparelhamento de diversos centros, com educação médica continuada, onde se pode oferecer mamografia adequada; maior número de diagnósticos da doença nos estádios precoces e, influenciando na redução da taxa de mortalidade para muIheres acima de 50 anos, em aproximadamente $30 \%$. Nesses locais, a taxa de diagnósticos de lesões avançadas tem sido inferior a $10 \%$ do total dos casos de câncer de mama ${ }^{7,13}$.

No Brasil, geralmente, 0 atendimento inicial da paciente com queixas mamárias é feito pelo médico ginecologista, o qual passa a ter um papel fundamental nas etapas para o diagnóstico da neoplasia. Entretanto, não se tem estabelecido através de estudo qual o conhecimento desses profissionais em relação ao diagnóstico do câncer de mama.
Dessa forma, o objetivo desse estudo foi avaliar o nível de conhecimento sobre o rastreamento e o diagnóstico do câncer de mama entre os ginecologistas do Estado de Goiás, além de verificar alguns dados pessoais e profissionais que pudessem predizer os profissionais com melhor conhecimento sobre o tema.

\section{Métodos}

Após aprovação pelo Comitê de Ética em Pesquisa do Hospital Araújojorge daAssociação de Combate ao Câncer em Goiás, foram enviados questionários a todos os ginecologistas inscritos naSociedade Goiana de Ginecologiae Obstetrícia entre maio e julho de 1999 . O questionário era constituído por perguntas objetivas sobre aspectos profissionais, bem como sobre o conhecimento a respeito do rastreamento e do diagnóstico do câncer de mama. $O$ questionário foi idealizado de forma que não houvesse identificação do médico que o respondeu (Tabela I). Junto com cada questionário, também foi enviado um envelope préselado, de modo a facilitar o retorno do mesmo. 
Tabela I - Questões abordadas e alternativas de respostas

I)Qual omelhor período para realizar oauto-exame?

a) Durantea menstruação*; b) Dois a cinco dias após a menstruação;c) No período ovulatório; d) No período pré-menstrual;e) Em qualquer dia do ciclo;ff Nãosei.

2)Qual o mais freqüente sinal ou sintoma do câncer de mama?

a) Mastalgia;b) Nódulo*;c)Descargamamilar; d) Linfonodos axilares;e) Espessamentoda pele;f) Nãosei.

3) Qual o melhor método pararastrear o câncer de mamaem mulheres assintomáticas?

a)Auto-exame;b) Mamografia*; c) Examefísico;d) Ultra-sonografia.

4)Quando indicar ouso da mamografia em mulheres assintomáticas?

a) 30 anos*; b) 40 anos; c) 50 anos; d)60 anos.

5)Qual a periodicidadeda mamografíapós os 50 anos?

a) Semestral;b)Anual*; c) Bienal; d) Trienal.

6)Qual a principal indicação do ultra-som associadoà mamografia na deteç̧ão do câncer de mama?

a) Emmamas volumosas; b) Emmamas pequenas; c) Emmamas densas/jovens*; E) Emmamas lipossubstituídas.

*Indicaaresposta correta paraa questão considerada.

Tabela 2 - Distribuição e frequêencia das respostas de acordo com as questões propostas

\begin{tabular}{|c|c|c|c|}
\hline \multirow[t]{2}{*}{ Pergunta } & \multicolumn{3}{|c|}{ Resposta } \\
\hline & $\begin{array}{c}\text { Correta } \\
\mathrm{n}(\%)\end{array}$ & $\begin{array}{l}\text { Incorreta } \\
\mathrm{n}(\%)\end{array}$ & $\begin{array}{c}\text { Total* } \\
\mathrm{n}\end{array}$ \\
\hline $\begin{array}{l}\text { Qual omelhor período pararealizar } \\
\text { oauto-exame? }\end{array}$ & $78(94)$ & $5(6)$ & 83 \\
\hline $\begin{array}{l}\text { Qual omaisfreqüente sinal ou sintoma } \\
\text { do câncer de mama? }\end{array}$ & $74(88)$ & $9(12)$ & 83 \\
\hline $\begin{array}{l}\text { Qual omelhor método para rastrear ocâncer } \\
\text { demamaemmulheres assintomáticas? }\end{array}$ & $61(75)$ & $20(25)$ & 81 \\
\hline $\begin{array}{l}\text { Quando indicar ouso damamografia } \\
\text { emmulheresassintomáticas? }\end{array}$ & $75(88)$ & $10(12)$ & 85 \\
\hline $\begin{array}{l}\text { Qual a periodicidade da mamografia } \\
\text { após os } 50 \text { anos? }\end{array}$ & 79(93) & $6(7)$ & 85 \\
\hline $\begin{array}{l}\text { Qual a principal indicação do ultra-som associadoà } \\
\text { mamografiana detecção do câncer demama? }\end{array}$ & $69(83)$ & $14(17)$ & 83 \\
\hline
\end{tabular}

Ototal de respostasédiferente entre as questões, visto que alguns médicos não responderam a todas elas.

Os questionários devolvidos foram arquivados e, posteriormente, os dados foram digitados em um banco de dados por um dos pesquisadores do estudo. Para a análise, foi determinada a freqüência das respostas corretas e, essas, cruzadas com informações pessoais e profissionais, incluindo: sexo, titularidade, portadores de Título de Especialista em Ginecologia e Obstetrícia (TEGO) e o local de atuação, as quais foram consideradas como variáveis independentes. Foram utilizados os testes qui-quadrado e t-Student quando cabíveis. Considerou-se o nível de significância de $95 \%(p<0,05)^{14}$.

\section{Resultados}

Dos 427 questionários enviados, 26 foram devolvidos por estarem com endereçamento incorretos. Dos 401 questionários endereçados corretamente, 85 médicos preencheram e retornaram os questionários, representando $21 \%$ do total dos enviados.

Em relação aos dados dos profissionais, $24(28 \%)$ eram do sexo feminino e 61 (72\%) do masculino. Com relação à titulação profissional, 60 (70\%) possuíam como maior titularidade a residência médica, 62 (73\%) eram portadores de TEGO. A média de idade dos ginecologistas que responderam foi de 44 anos.
A freqüência e distribuição das respostas corretas estão indicadas na Tabela 2.

Quando questionados sobre o conhecimento em câncer de mama, I,2\% dos ginecologistas consideraram possuir um ótimo conhecimento sobre o câncer de mama, $20 \%$ um conhecimento muito bom, $50,6 \%$ acreditavam ter um bom nível de conhecimento, $22,4 \%$ regular eapenas $5,8 \%$ considerava ter um fraco conhecimento. Todos os profissionais referiram ter conhecimento da técnica de auto-exame, porém, dentre as mulheres, $8 \%$ não praticam o auto-exame das mamas, tendo como motivo o fato de considerarem que não serão acometidas pela doença.

Sobre a freqüência em que os profissionais realizam o exame físico das mamas das pacientes, $94 \%$ dos médicos o fazem sempre, $3,5 \%$ realizam apenas quando há queixas mamárias, e I\% quando selembram. Os demais só fazem o exame se a paciente solicitar.

Também foi questionada qual seria a conduta do profissional frente a um nódulo palpável. A maioria respondeu que prefere iniciar a investigação armada por ele próprio. Desta forma, a punção aspirativa por agulha fina é preferida por $56,6 \%$ dos ginecologistas, seguido de $19,3 \%$ que preferem realizar biópsia aberta, além de $6 \%$ que fazem a punção histológica por agulha grossa. Apenas $18,1 \%$ dos profissionais preferem encaminhar a paciente para um colega treinado em doenças mamárias, assim que detectada a lesão.

As variáveis independentes foram cruzadas com as questões propostas. Com relação ao sexo, não houve influência na taxa de respostas corretas (Tabela 3).

Em relação à titularidade, o fato do profissional ter cursado um programa de residência médica influenciou sobre a taxa de respostas corretas. Houve um número de respostas corretas significativamente maior no questionamento sobre a associação da ultra-sonografia à mamografia ( $90 \%$ versus $58 \% ; p=0,00 \mathrm{I}$ ) e na indicação de realização da mamografia ( $92 \%$ versus $75 \%$; $p=0,05$ ), conforme mostrado na Tabela 4.

$O$ fato de o profissional ter obtido 0 TEGO não influenciou a taxa de respostas corretas (Tabela 5). O local de atuação profissional, se na capital ou no interior de Goiás, também não influenciou a taxa de acerto das questões (Tabela 6). 


\begin{tabular}{lccc}
\hline \multicolumn{3}{c}{ Tabela 3 - Conhecimento do diagnóstico do câncer de mama entre os ginecologistas } \\
de Goiás de acordo com o sexo
\end{tabular}

Tabela 4 - Conhecimento do diagnóstico do câncer de mama entre os ginecologistas de Goiás de acordo com a titularidade

\begin{tabular}{lccc}
\hline \multirow{2}{*}{ Pergunta } & \multicolumn{3}{c}{ Residência Médica } \\
\cline { 2 - 4 } & Sim & Não & \\
& $n(\%)$ & $n(\%)$ & $\mathrm{P}$ \\
Melhorperíodopararealizarauto-exame & $57(97)$ & $17(89)$ & 0,24 \\
Sinal ou sintoma maisfreqüente & $54(91)$ & $16(80)$ & 0,16 \\
Melhormétodo pararastreamento & $45(76)$ & $11(65)$ & 0,34 \\
Indicaçãodamamografia & $55(92)$ & $15(75)$ & $0,05 *$ \\
Periodicidadedamamografia & $56(93)$ & $18(20)$ & 1,0 \\
Indicaçãodoultra-somassociado & $53(90)$ & $11(58)$ & $0,00 I^{*}$ \\
\hline
\end{tabular}

* representadiferenças estatisticamente significantes entre os grupos.

Tabela 5 - Conhecimento do diagnóstico do câncer de mama entre os ginecologistas de Goiás de acordo com obtenção de TEGO

\begin{tabular}{lccc}
\hline Pergunta & \multicolumn{3}{c}{ TEGO } \\
\cline { 2 - 4 } & Sim & não & \\
Melhor período para realizaroauto-exame & $n(\%)$ & $n(\%)$ & $\mathrm{P}$ \\
Sinal ou sintoma maisfreqüente & $57(93)$ & $20(95)$ & 0,82 \\
Melhor método para rastreamento & $55(90)$ & $18(82)$ & 0,30 \\
Indicaçãodamamografia & $43(73)$ & $17(81)$ & 0,46 \\
Periodicidadedamamografia & $57(92)$ & $18(82)$ & 0,18 \\
Indicaçãodoultra-somassociado & $57(92)$ & $22(100)$ & 0,40 \\
& $51(84)$ & $17(81)$ & 0,78 \\
\hline
\end{tabular}

Tabela 6 - Conhecimento do diagnóstico do câncer de mama entre os ginecologistas de Goiás de acordo com o local de atuação

\begin{tabular}{lccc}
\hline \multirow{2}{*}{ Pergunta } & \multicolumn{3}{c}{ Local de atuação } \\
\cline { 2 - 4 } & Goiânia & Interior \\
& $\mathrm{n}(\%)$ & $\mathrm{n}(\%)$ & $\mathrm{p}$ \\
Melhor período para realizar oauto-exame & $46(96)$ & $31(94)$ & 0,89 \\
Sinal ousintomamaisfreqüente & $42(89)$ & $30(86)$ & 0,61 \\
Melhor método pararastreamento & $33(70)$ & $26(81)$ & 0,26 \\
Indicaçãodamamografia & $43(89)$ & $30(86)$ & 0,59 \\
Periodicidadedamamografia & $46(96)$ & $31(89)$ & 0,40 \\
Indicaçãodoultra-somassociado & $39(83)$ & $28(82)$ & 0,94 \\
\hline
\end{tabular}

\section{Discussão}

Em estudo prévio, identificamos que é grande o desconhecimento a respeito do câncer de mama nas diversas camadas da nossa sociedade ${ }^{8}$. Considerando que o profissional médico é o principal responsável como informante e esclarecedor da população, seja no trabalho corpo a corpo desenvolvido nos consultórios e ambulatórios, seja através dos meios de comunicação de massa ou de campanhas preventivas, tornou-se necessário um estudo a respeito do conhecimento dos nossos profissionais no tocante aos métodos de prevençãoe diagnóstico.

Em recente estudo sobre o conhecimento do diagnóstico do câncer de mama entre estudantes de Medicina, desenvolvido na UFG, observou-se que os acadêmicos do $5^{\circ}$ ano médico tinham melhor conhecimento dos métodos diagnósticos que os acadêmicos do $1{ }^{\circ}$ ao $4^{\circ}$ ano, os quais não haviam cursado a disciplina de Ginecologia. Oitenta por cento dos alunos do $5^{\circ}$ ano sabiam quando indicar a mamografia e $71 \%$ tinham conhecimento da periodicidade do exame após os 50 anos, mas boa parte destes alunos ainda não tinha um adequado conhecimento a respeito de questionamentos simples, como o melhor método de rastreamento do câncer de mama e quando associar a ultra-sonografia ao exame mamográfico. Ainda neste estudo, questionou-se sobre os métodos de divulgação a respeito do diagnóstico do câncer de mama e $63 \%$ dos alunos os julgaram inadequados e insuficientes ${ }^{15}$.

Como ainda existem falhas na formação médica, é possível que isso seja extensivo aos profissionais já engajados no mercado de trabalho e que lidam diretamente com a patologia mamária. Tem sido divulgado, ocasionalmente, pela mídia que os profissionais da área de saúde não estariam adequadamente preparados para o reconhecimento e abordagem das doenças da mama, principalmente do câncer de mama. Entretanto, os dados apresentados no presente trabalho contrapõem a essa idéia uma vez que a taxa de respostas corretas, para cada um dos questionamentos a respeito do diagnóstico do câncer de mama, foi bastante alta (todas acima de $75 \%$ ). Esse fato não pode ser estabelecido na íntegra, uma vez que somente $21 \%$ dos questionários enviados foram retornados para a análise do estudo. É possível 
que os médicos que tenham retornado seus formulários sejam aqueles que demonstrem maior interesse pela profissão e pela especialidade, podendo ter levado a um viés no estudo, favorecendo a alta taxa de acerto para os quesitos propostos.

Deve ser observado que não houve diferenças significativas de acerto para as respostas quando comparamos se os profissionais eram do sexo masculino ou feminino.

Para nossa surpresa, também não houve diferença na parte de respostas corretas entre os profissionais que são possuidores do título de especialista em Ginecologia e Obstetrícia ou não e também para aqueles profissionais que atuam em Goiânia (capital) ou no interior do Estado de Goiás. Acreditávamos que os possuidores de TEGO e os profissionais que militam na capital, pudessem ter um acesso maior à informação referente ao rastreamento do diagnóstico do câncer de mama e, assim, apresentar uma maior taxa de respostas corretas.

Das variáveis estudadas, aquela que interferiu na taxa de acertos foi em relação aos profissionais que fizeram residência médica, ao serem comparados com aqueles que não cursaram tal programa. Os profissionais com residência médica em Ginecologia tiveram um número de respostas corretas significativamente maior que os que não cursaram, em relação à indicação da mamografia $(92 \% \mathrm{x}$ $75 \%$, respectivamente; $p=0,05)$ e da indicação do ultra-som associado à mamografia ( $90 \% \times 58 \%$, respectivamente; $p=0,00 \mathrm{I})$. Esse fato já era esperado, uma vez que os profissionais que cursam os programas de residência médica têm acesso a um maior volume de informações do que os outros que não tiveram possibilidade de cursar tais programas.

No tocante ao conhecimento sobre 0 diagnóstico do câncer de mama, metade dos profissionais acredita ter bom conhecimento sobre o tema e apenas $7 \%$ da população estudada se considerava fraco ou tendo conhecimentos regulares a esse respeito.

Esse fato reflete a segurança dos profissionais estudados, visto que $56 \%$ deles já façam por si mesmos a abordagem do nódulo através de punção aspirativa por agulha fina, além de outros $25 \%$ que utilizam métodos mais agressivos, como biópsias incisionais ou excisionais na sua prática diária.
É importante lembrar que $8 \%$ das médicas não realizam o auto-exame das mamas, mesmo detendo todo o conhecimento sobre o diagnóstico, desenvolvimento, tratamento e prognóstico da doença. Esse número torna-se bastante expressivo por ser considerado dentro de uma classe esclarecida sobre $o$ assunto e que, entre as mulheres em geral, esse número seja bem maior, em razão da falta de conhecimento da população.

Assim, concluímos pelo presente estudo que, dos médicos que encaminharam seus questionários respondidos, houve uma alta taxa de respostas corretas para todos os quesitos sobre o diagnóstico do câncer de mama, refletindo nesse grupo um alto conhecimento sobre o tema, o qual foi apenas influenciado, ou melhorado, por aqueles profissionais que cursaram programa de residência médica.

\section{SUMMARY

$\begin{array}{lccr}\text { KNOWLEDGE } & \text { ON } & \text { SCREENING } & \text { AND } \\ \text { DIAGNOSIS OF } & \text { BREAST } & \text { CANCER } & \text { AMONG } \\ \text { GYNECOLOGISTS } & \text { FROM } & \text { G OIÁS } & \text { STATE } \\ \text { (BrAZIL) } & & & \end{array}$

Objectives. To evaluate the knowledge level on the screening and diagnosis of breast cancer among the gynecologists of the State of Goiás, Brazil.

METHODS. Questionnaires containing professional information and questions on breast cancer screening were posted for all the 427 gynecologists associated to the Goiás Society of Gynecology and Obstetrics, between May and July of 1999.

RESULTS. Of the 427 questionnaires, 26 (6\%) were returned because incorrect mailing address. Of the 401 doctors who received the questionnaire, $85(21 \%)$ answered and returned them back. Of these, $28 \%$ were female; the average of the profession time was of 17 years. The medical residence was the highest title level for $70 \%$. The average age of the doctors was 44 years. The rates of correct answers were: $94 \%$ for the knowledge of the most frequent sign of breast cancer, $75 \%$ for the best method for screening, $88 \%$ for the period of when the mammography begins, $93 \%$ for the periodicity of the mammography after the 50 years and $83 \%$ about the indication of the complementary ultrasound. The doctors who have studied in a program of medical residence was the only factor that influenced the rate of correct answers, if compared to those that did not cursed it. The professional's gender, the Title in Gynecology and Obstetrics Specialty (TEGO) holders and the city of work did not influence the rates of correct answers.

CONCLUSION. The gynecologists of the State of Goiás who answered the questionnaire have a good knowledge on the screening and diagnosis of breast cancer. Medical residence was the single factor that influenced the rate of correct answers. [Rev Assoc Med Bras 2003; 49(3): 312-6]

KEYwords: Breast. Cancer. Diagnosis. Medical education. Gynecologist.

\section{ReFERÊNCIAS}

I. Coleman MP, Sloggett A, Babb P, Kaplan RM, Wingard DL. Trends in breast cancer incidence, survival and mortality. Lancet 2000; 356: 590-59l.

2. Parkin D. Global cancer statistics in the year 2000. Lancet Oncol 2001 ; 2:533-43.

3. Ries L, Eisner M, Kosary C, Howe HL, Wingo PA, Thun MJ. SEER Cancer Statistics Review, 1973-1998. Bethesda: National Cancer Institute; 2001 .

4. Paulinelli RR, Freitas Jr R, Curado MP, Souza AA. A situação do câncer de mama em Goiás, no Brasil e no Mundo: tendências atuais para a incidência e mortalidade. Rev Bras Saúde Matern Infantil 2003; 3: I 7-24.

5. Brasil. Ministério da Saúde. Sistema Único de Saúde. Instituto Nacional de Câncer - INCA. Estimativas da incidência e mortalidade por câncer. Rio de Janeiro: INCA; 200I.

6. Pascalicchio JC, Fristachi CE, Baracat FF. Câncer de mama: fatores de risco, prognósticos e preditivos. Rev Bras Mastol 200I; I I:7I-84.

7. Sant M, Capocaccia R, Coleman MP, Berrino F, Gatta G, Micheli A et al. Cancer survival increases in Europe, but international differences remain wide. Zapponeb and the EUROCARE Working Group I. Eur J Cancer 200 I; 37: I659-67.

8. Silveira Jr LP, Freitas Jr R, Carneiro AB, Ribeiro LFJ, Queiroz GS. Fatores sócio-demográficos associados com o estadiamento das pacientes com câncer de mama. Rev Bras Ginecol Obstet 1996; I8:4 I I-5.

9. Montella M, Biondi E, Marco MD, Botti G, Fatangelo F, Capasso let al. Sociodemographic factors associated with the diagnostic staging of breast cancer in Southern Italy. Cancer 1995; 76: 1585-90.

I0. FreitasJr R, Paulinelli R, CoelhoA, Ribeiro LFJ, Queiroz GS, Esperidião MD et al. Análise da sobrevida de pacientes com câncer de mama localmente avançado submetidas à quimioterapia neo-adjuvante (FACV). Rev Bras Mastol 1997; 7:9-15. 
1I. Caldeira J, Budin R. Aspectos epidemiológicos do câncer de mama em Jaú-SP e a elevada incidência de casos avançados em mulheres idosas. Rev Bras Cancerol 1995; 41:15-7.

12. CezarJr O. Carcinoma de mama em Bragança Paulista. Experiência de uma década. GO Atual 1996; 8:9-13

13. Kerlikowske K, Grady D, Rubin SM, Sandrock
C, Ernster VL. Efficacy of screening mammography. A meta analysis. JAMA 1995 , 273: 149-54.

14. ROUQUAYROL, M.Z. Epidemiologia e saúde. $3^{\text {a }}$ ed. Rio de Janeiro:MEDSI Editora Científica; 1988.

15. Aires NM, Freitas Jr R, Paulinelli RR, Baêta LF, Finotti MCCF, Silveira MT. Conhecimento dos métodos diagnósticos do câncer de mama entre estudantes de medicina. Rev Bras Ginecol Obstet 1999: 21:133-7.

Aceito para publicação: 29/05/03

\section{IMAGEM EM MEDICINA}

Participe da nova seção da Ramb. É um espaço aberto ao leitor, que poderá enviar material de interesse educativo como fotos, ilustrações e exames, acrescido de três linhas explicativas contendo ainda nome do autor e serviço onde foi realizado. O material poderá ser enviado para a

Rua São Carlos do Pinhal, 324 - Cep 01333-903 S. Paulo - SP - E-mail: ramb@amb.org.br 